\title{
Editorial: Smart Devices \& Smart Spaces in Wireless Internet of Everything (Wireless-IoE)
}

\author{
Donghwi Lee ${ }^{1}$ Kyong-ho $\mathrm{Choi}^{2} \cdot \mathrm{Hyuncheol} \mathrm{Kim}^{3}$
}

Published online: 10 April 2017

(C) Springer Science+Business Media New York 2017

Internet of Everything (IoE) is based on Internet of things (IoT), but in addition to IoT, it also includes other technical innovations such as cloud, big data, and IPv6. Cisco defines the age of IoE as "an era in which unprecedented value is created by real-time interaction with people, processes, data, things, and the rest of the unconnected world, all connected to the Internet." In other words, if IoT is technology, IoE can be called "future lifestyle" or "lifestyle innovation", which will be different from current one through real-time connectivity rather than simply a collection of technologies or technologies.

The Wireless Internet of Everything (Wireless-IoE) is a novel paradigm that is shaping the evolution of the future ubiquitous networks. According to the vision underlying the IoE, the next step in increasing the ubiquity of the Internet is to connect smart devices for smart spaces. By providing a highly distributed and ubiquitous network of seamlessly connected heterogeneous smart devices, new services for smart space will be designed to serve anytime, anywhere, and by anyone and anything. "Smart Devices \& Smart Spaces in Wireless Internet of Everything (Wireless-IoE)" is an important multidisciplinary area with high social and business impact fall under the IoE, including personal healthcare, smart cloud, smart city, smart home, and smart transportation, and it is strongly expected that new applications will emerge once the enabling technologies reach a stable state. However, various studies have been conducted on Wireless-IoE, but there are still many problems to be solved.

Donghwi Lee

dhclub@dsu.ac.kr

Kyong-ho Choi

cyberckh@jftech.kr

Hyuncheol Kim

hckim@nsu.ac.kr

1 Dongshin University, Naju, South Korea

2 R\&D Center, JF Tech, Seoul, South Korea

3 Nam Seoul University, Cheonan, South Korea 
This special issue included ten carefully selected papers and covers high-quality and state-of-the-art research issues and results regarding the Smart Devices \& Smart Spaces in Wireless-IoE techniques.

Four papers discuss security issues in wireless-IoE. Kim et al.'s paper proposes a customized user interface using a network-based interface for the cloud as the optimized interface with a variety of individual application environments. Minsu Kim and Kuinam J. Kim propose a system for integrity security patch updating using PM-FTS (Patch Management-File Transfer System). Hu et al.'s paper proposes an iterative algorithm to carry out energy-balanced vector modulation. Park et al.'s paper compares Snort and Suricata's processing and detection rate to decide which is better in single threading or multithreading environment.

Three papers deal with various basic research for IoE services. Baek et al.'s paper propose ARINC661 rendering based on OpenVG. Lee et al.'s paper proposes an object tracking and recognition system according to reliability assessment of learning in mobile environments. Jong Chan Lee et al.'s paper proposes resource reservation scheme for the handoff control to accommodate mobile multimedia traffic using the location estimation and the direction estimation.

Finally, the remaining three papers describe various applications and services of wireless-IoE. Setthawong et al.'s paper proposes alternative approaches that can provide the perception of 3D and depth to the visual presentation with less strain on the eyes. Nagoya et al.'s paper describes a development of desktop application software by connecting with several software components. Sang-Hyun Lee and Sang-Joon Lee's paper suggests a neural network model will be superior to the best traditional diffusion model.

At last, guest editors would like to take this opportunity to thank all the authors of the special issue for their excellent contributions, and reviewers, for their treasurable comments. Springer publishing staff is also acknowledged for their kindness, swiftness, and enthusiasm for this issue.

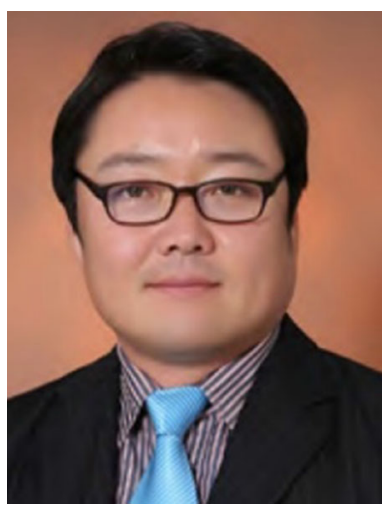

Donghwi Lee received the Ph.D. degree in Information Security from Kyonggi University, Korea, in 2001 and 2007. Research Scholar of the University of Colorado Denver in 2010 and 2011, USA, He is currently a Professor of DongShin University, His research areas include Information Security and Control System Security. 

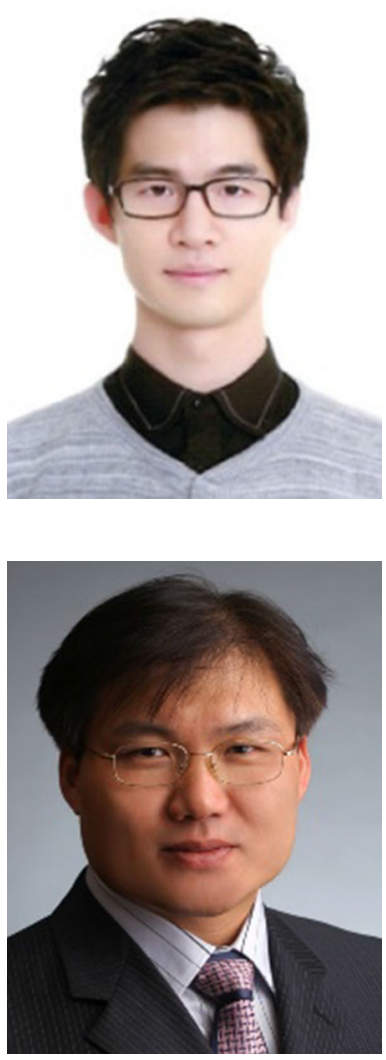

Kyong-ho Choi received the B.S. and M.S. degrees from Dept. of Economy, and a Ph.D. degree from Dept. of Information Security, Kyonggi University, Korea, in 2002, 2005 and 2008. He is currently a Head of R\&D Center of JF Tech, Korea. His research interests include the economics of information security, service science, and convergence security.

Hyuncheol Kim received his Ph. D. degree in the department of information engineering from Sungkyunkwan University, Korea, in 2005. From 1992 to 2002, he was a senior engineer in Electronics and Telecommunications Research Institute (ETRI), Daejeon, Korea. He has been a professor in the department of computer science at Namseoul University, Korea. His research interests include cloud computing, next generation network and embedded computing systems. 